\title{
Clinical characteristics and prognosis of elderly nasopharyngeal carcinoma patients receiving intensity-modulated radiotherapy
}

\author{
Yingchen Lyu ${ }^{1,2} \cdot$ Mengshan $\mathrm{Ni}^{1,2} \cdot$ Ruiping $\mathrm{Zhai}^{1,2} \cdot$ Fangfang Kong ${ }^{1,2} \cdot$ Chengrun $\mathrm{Du}^{1,2} \cdot$ Chaosu $\mathrm{Hu}^{1,2}$. \\ Hongmei Ying ${ }^{1,2}$ (D)
}

Received: 10 June 2020 / Accepted: 23 September 2020 / Published online: 6 October 2020

(c) The Author(s) 2020

\begin{abstract}
Purpose To evaluate the clinical characteristics and prognosis of elderly nasopharyngeal carcinoma (NPC) patients receiving intensity-modulated radiotherapy (IMRT).

Methods From June 2008 to October 2014, 148 newly diagnosed non-metastatic elderly NPC patients (aged $\geq 70$ years) receiving IMRT were recruited. Comorbid condition was evaluated using the age-adjusted Charlson Comorbidity Index (ACCI). Kaplan-Meier method was used to estimate survival rates and the differences were compared using log-rank test. Hazard ratio (HR) and the associated 95\% confidence interval (CI) were calculated using Cox proportional hazard model by means of multivariate analysis.

Results The median follow-up time was 66.35 months. Estimated OS rate at 5 years for the entire group was $61.8 \%$ (95\% confidence interval [CI] 0.542-0.703). The 5-year OS rate of RT alone group was 58.4\% (95\% [CI] 0.490-0.696) compared with $65.2 \%$ (95\% [CI] 0.534-0.796) in CRT group $(p=0.45)$.

In patients receiving IMRT only, ACCI score equal to 3 was correlated with superior 5-year OS rate in comparison with higher ACCI score $62.1 \%$ (95\% [CI] 0.510-0.766) to 48.5\% (95\% [CI] 0.341-0.689), respectively; $p=0.024)$. A 5-year OS rate of $63.1 \%$ (95\% [CI] 0.537-0.741) was observed in patients younger than 75 years old compared with $57.5 \%$ (95\% [CI] $0.457-0.723)$ in patients older $(p=0.026)$. Patients with early-stage disease (I-II) showed better prognosis than patients with advanced-stage (III-IV) disease (5-year OS, 72.3-55.4\%, respectively; $p=0.0073$ ). The Cox proportional hazards model suggested that age independently predicted poorer OS (HR, 1.07; 95\%CI 1.00-1.15, $p=0.04$ ).

Conclusion The survival outcome of patients aged $\geq 70$ years receiving IMRT only was similar to chemoradiotherapy with significantly less acute toxicities. Among the population, age is significantly prognostic for survival outcomes.
\end{abstract}

Keywords Age-adjusted Charlson comorbidity index $\cdot$ Nasopharyngeal carcinoma $\cdot$ Intensity-modulated radiotherapy · Elderly

Yingchen Lyu and Mengshan Ni contributed equally to this work.

Hongmei Ying

Hongmeiying111@163.com

1 Department of Radiation Oncology, Fudan University Shanghai Cancer Center, 270 Dong'an Road, Shanghai 200032, China

2 Department of Oncology, Shanghai Medical College, Fudan University, Shanghai, China

\section{Introduction}

Nasopharyngeal carcinoma is characterized by its unique and extremely unbalanced geographical distribution, with $70 \%$ cases in the east and Southeast Asia [1]. Unlike the bimodal distribution in low-risk populations, the age distribution in epidemic area peaks in individuals aged 45-59 [2, 3]. As the percentage of elderly people is gradually increasing globally, the occurrence of NPC in the elderly is not rare. Despite the heavier health burden of the geriatric population, elderly individuals have been underrepresented in clinical studies. Particularly, those aged $\geq 70$ years attributed less than $5 \%$ in prospective trials in head and neck cancer [4]. In MAC-NPC meta-analysis, those aged $\geq 60$ years constituted merely $13 \%$ 
of the total cohort [5]. The proportion of $\geq 70$ years age was likely less than half of that figure. The treatment paradigms in the elderly NPC patients have not been well defined for the limited researches.

Opinions were divided on whether standard aggressive treatment can bring benefit to this vulnerable population. Some argued that elderly NPC patients should receive chemotherapy for improved survival outcome [6-8]. In those mentioned studies, most of the patients were treated with conventional radiotherapy. It was not clear that adding chemotherapy could bring benefit to patients receiving IMRT, which has now been widely used with excellent locoregional control in NPC. Moreover, selecting 60-65 as the cut-off value in those studies for the elderly may not be reasonable for the improved physical health of this population. Patients aged $\geq 70$ years were closely associated with multiple comorbidities, poor performance status and reduced organ reserve, which accounted for their lower tolerance and severe toxicity for chemotherapy $[9,10]$. Thus, tailored and less aggressive treatment strategies in elderly individuals should be considered.

In this article, we focused on the survival and prognosis of senior NPC patients receiving Intensity-Modulated radiotherapy and assessed the comorbidities utilizing ageadjusted Charlson Comorbidity Index.

\section{Materials and methods}

\section{Patient characteristics}

This retrospective study received approval from the Institutional Review Board. The document requirement was waived because of the retrospective nature of the study. The research presented no more than minimal risk with only clinical and dosimetric data studied.

Between June 2008 and October 2014, a total of 148 NPC patients undergoing IMRT at Fudan University Shanghai Cancer Center (FUSCC) were included in this study. The eligible criteria were: (1) age of 70 years old and above; (2) histologically proven NPC; (3) treated with IMRT. The exclusion criteria were: (1) presence of distant metastasis; (2) underwent surgery; (3) history of head and/or neck irradiation; (4) Karnofsky Performance Score $<70$.

\section{Clinical staging and co-morbidity assessment}

Pretreatment assessment consisted of complete patient history, thorough physical examination, hematology and biochemistry profiles, nasopharyngeal magnetic resonance imaging (MRI), neck MRI or computed tomography (CT), bone scan, chest $\mathrm{X}$-ray or $\mathrm{CT}$, abdominal ultrasound. All patients underwent restaging according to the eighth edition guideline of the American Joint Commission on Cancer staging. Considering the vulnerable nature of the studied population, comorbid conditions were evaluated using the age-adjusted Charlson Comorbidity Index (ACCI), which has been shown to be an independent predictor of long-term survival.

\section{Radiotherapy}

Patients were immobilized in the supine position with a thermoplastic mask. CT was performed after immobilization, obtaining 5-mm slices from the anterior clinoid process $2 \mathrm{~cm}$ below the sternoclavicular joint. All the patients received IMRT with six megavoltage photons (6MV). In brief, the total dose was 66-70.4 Gy in 30-35 fractions to primary lesion of nasopharynx, 66 or $70.4 \mathrm{~Gy}$ in $30-35$ fractions to metastatic lymph nodes of the neck, 60 Gy to high-risk CTV and 54 Gy to low-risk CTV, respectively. Small-field IMRT was applied to treat local residual disease just after the planned treatment with 2.2-4.4 Gy in one or two fractions. Residual nodes were treated with a boost of 4-6 Gy in 2 or 3 daily fractions using an electron field of 9-12 meV just after the planned treatment.

\section{Follow-up and statistical analysis}

During radiotherapy, patients underwent assessment weekly. After completing radiotherapy, patients were followed up every 3 months in the first 2 years, and then every 6 months from year 2 to year 5 , and annually thereafter. Survival time was measured from the initiation of the RT to date of death or the latest date of follow-up for patients still alive.

Kaplan-Meier method was used to estimate survival rates and the differences were compared using log-rank test. Hazard ratio (HR) and the associated $95 \%$ confidence interval (CI) were calculated using Cox proportional hazard model. The $\chi^{2}$ test was used for comparing categorical variables, and independent $t$-test was used for comparing the means of continuous variables. Covariates including age, sex, age-adjusted Charlson Comorbidity Index, $N$ category, $T$ category, overall stage, radiation dose, and chemotherapy were included in all tests. Cox proportional hazards model was performed to carry out univariate analysis and multivariate analysis. A two-sided $p$ value of $<0.05$ was considered statistically significant. All data analyses and drawings were completed using R software 4.0.0 (https://www.r-project.org).

\section{Results}

\section{Baseline characteristics}

Table 1 demonstrated the patients' characteristics. Median age was 74.18 years (age ranged $70-93$ years). The ratio of male to female was $3.48: 1$, with 115 males and 33 females. According to the AJCC/UICC (8th edition) staging criteria, 
there were $26(17.6 \%)$ patients with stage I disease, 53 (35.8\%) patients with stage II disease, 31 (20.9\%) patients with stage III disease and $28(18.9 \%)$ patients with stage IV disease. The comorbidity level was sored according to the ACCI. The score was 3 in 105 (70.9\%), 4 in $27(18.2 \%), 5$ in $12(8.1 \%), 6$ in $2(1.4 \%), 7$ in $1(0.7 \%)$ and 13 in $1(0.7 \%)$. 89 patients received IMRT only. Induction chemotherapy was applied to 44 (27.2\%) with concurrent chemotherapy in $27(18.2 \%)$ and adjuvant chemotherapy in $6(4.1 \%)$.

Table 1 Baseline characteristics of patients

\begin{tabular}{|c|c|}
\hline Level & No. of patients \\
\hline$n$ & 148 \\
\hline Age [mean (SD)] & $74.18(3.86)$ \\
\hline \multicolumn{2}{|l|}{ Gender $(\%)$} \\
\hline Female & $33(22.3)$ \\
\hline Male & 115 (77.7) \\
\hline \multicolumn{2}{|l|}{ ACCI (\%) } \\
\hline 3 & 105 (70.9) \\
\hline 4 & $27(18.2)$ \\
\hline 5 & $12(8.1)$ \\
\hline 6 & $2(1.4)$ \\
\hline 7 & $1(0.7)$ \\
\hline 13 & $1(0.7)$ \\
\hline \multicolumn{2}{|l|}{ Treatment (\%) } \\
\hline RT alone & 89 (60.1) \\
\hline Induction CT & $44(27.2)$ \\
\hline Concurrent $\mathrm{CT}$ & $27(18.2)$ \\
\hline Adjuvant CT & $6(4.1)$ \\
\hline \multicolumn{2}{|l|}{$T(\%)$} \\
\hline 1 & $26(17.6)$ \\
\hline 2 & $53(35.8)$ \\
\hline 3 & $31(20.9)$ \\
\hline 4 & $38(25.7)$ \\
\hline \multicolumn{2}{|l|}{$N(\%)$} \\
\hline 0 & $28(18.9)$ \\
\hline 1 & $67(45.3)$ \\
\hline 2 & $39(26.4)$ \\
\hline 3 & $14(9.5)$ \\
\hline \multicolumn{2}{|l|}{ 8th AJCC stage (\%) } \\
\hline 1 & $11(7.4)$ \\
\hline 2 & $37(25.0)$ \\
\hline 3 & $52(35.1)$ \\
\hline 4 & $48(32.4)$ \\
\hline \multicolumn{2}{|l|}{ Radiation dose (\%) } \\
\hline$\leq 6600 \mathrm{~Gy}$ & $75(50.7)$ \\
\hline$>6600 \mathrm{~Gy}$ & $73(49.3)$ \\
\hline Follow-up time (months) (median [IQR]) & $66.35[29.03,86.74]$ \\
\hline
\end{tabular}

$A C C I$ age-adjusted Charlson Comorbidity Index, AJCC American Joint Commission on Cancer staging, $C T$ chemotherapy, IQR InterQuartile Range
Patients receiving radiotherapy alone tended to have older age (median age 75.24 vs.72.58) $(p<0.001)$, earlier stage $(p<0.001)$ and higher ACCI (Table 2).

\section{Radiotherapy course and toxicity}

In all, 144 patients (97.3\%) completed the planned RT. Of four who failed, one patient received the total dose $<60 \mathrm{~Gy}$ because of treatment toxicities, two patients were administrated with $60 \mathrm{~Gy}$ because of their own will. The median actuarial irradiated dose of the nasopharynx was $66 \mathrm{~Gy}$ (range 35.2-74.4 Gy), and 10 patients (6.7\%) received a boost for residual primary disease.

The effect of acute toxicity in radiotherapy (RT) alone and chemoradiotherapy (CRT) group was shown in Table 3 . The rates of severe mucositis and dermatitis were higher in CRT group. Besides, CRT group developed significantly higher leukopenia, Neutrocytopenia, Thrombocytopaenia $(p<0.001)$ compared to RT alone group. In RT alone group, the majority developed with grade 1 to 2 toxicity. Severe acute mucositis and dermatitis (grade 3 or 4 ) occurred in $24.72 \%$ and $5.62 \%$, respectively. Emesis occurred in $11.2 \%$ of patients without grade 3 or 4 events. For hematologic adverse events, incidence of grade 3 leukopenia was $1.12 \%$, which was the same with incidence of grade 3 neutrocytopenia and thrombocytopenia. No grade 4 hematologic toxicity was observed. Details of the acute toxicity are listed as Table 3.

\section{Prognostic factors and survival}

The median follow-up time was 66.35 months. Estimated OS rate at 5 years for the entire group was $61.8 \%$ (95\% confidence interval [CI] 0.542-0.703). The addition of chemotherapy to IMRT failed to enhance the survival outcomes of patients. The 5-year OS rate of RT alone group was $58.4 \%(95 \%[\mathrm{CI}]$ 0.490-0.696) compared with $65.2 \%$ (95\% [CI] 0.534-0.796) in CRT group $(p=0.45)$ (Fig. 1).

In patients receiving IMRT only, ACCI score equal to 3 was correlated with superior 5-year OS rate in comparison with higher ACCI score $62.1 \%$ (95\% [CI] 0.510-0.766) to 48.5\% (95\% [CI] 0.341-0.689), respectively; $p=0.024$ ) (Fig. 2a). A 5 -year OS rate of $63.1 \%$ (95\% [CI] 0.537-0.741) was observed in patients younger than 75 years old compared with $57.5 \%$ (95\% [CI $0.457-0.723)$ in patients older $(p=0.026)$ (Fig. 3a). In patients receiving RT only, the overall survival of aged $\leq 75$ was also superior to the elder counterpart $(p=0.032)$ (Fig. 3b). Patients with early-stage disease (I-II) showed better prognosis than patients with advanced-stage (III-IV) disease (5-year OS, $72.3 \%$ to $55.4 \%$, respectively; $p=0.0073$ ) (Fig. 4 a). In patients receiving RT only, prognosis of early-stage was also more favorable than advanced-stage diseases $(p=0.00022)$ (Fig. 4b). However, the positive association of radiation dose with OS in both groups was not found. 
Table 2 Comparation of baseline characteristics in RT alone and CRT group

\begin{tabular}{|c|c|c|c|}
\hline Level & RT & CRT & $p$ \\
\hline$n$ & 89 & 59 & \\
\hline Age [mean (SD)] & $75.24(4.21)$ & $72.58(2.59)$ & $<0.001$ \\
\hline \multicolumn{4}{|l|}{ Gender $(\%)$} \\
\hline 0 & $22(24.7)$ & $11(18.6)$ & \multirow[t]{2}{*}{0.426} \\
\hline 1 & $67(75.3)$ & $48(81.4)$ & \\
\hline \multicolumn{4}{|l|}{ ACCI (\%) } \\
\hline 3 & $56(62.9)$ & $49(83.1)$ & \multirow[t]{6}{*}{0.117} \\
\hline 4 & $20(22.5)$ & 7 (11.9) & \\
\hline 5 & $9(10.1)$ & $3(5.1)$ & \\
\hline 6 & $2(2.2)$ & $0(0.0)$ & \\
\hline 7 & $1(1.1)$ & $0(0.0)$ & \\
\hline 13 & $1(1.1)$ & $0(0.0)$ & \\
\hline \multicolumn{4}{|l|}{$T(\%)$} \\
\hline 1 & $23(25.8)$ & $3(5.1)$ & \multirow[t]{4}{*}{0.001} \\
\hline 2 & $34(38.2)$ & $19(32.2)$ & \\
\hline 3 & $15(16.9)$ & $16(27.1)$ & \\
\hline 4 & $17(19.1)$ & $21(35.6)$ & \\
\hline \multicolumn{4}{|l|}{$N(\%)$} \\
\hline 0 & $23(25.8)$ & $5(8.5)$ & \multirow[t]{4}{*}{0.001} \\
\hline 1 & $45(50.6)$ & $22(37.3)$ & \\
\hline 2 & $16(18.0)$ & $23(39.0)$ & \\
\hline 3 & $5(5.6)$ & $9(15.3)$ & \\
\hline \multicolumn{4}{|l|}{ 8th AJCC stage (\%) } \\
\hline 1 & $11(12.4)$ & $0(0.0)$ & \multirow[t]{4}{*}{$<0.001$} \\
\hline 2 & $31(34.8)$ & $6(10.2)$ & \\
\hline 3 & $25(28.1)$ & $27(45.8)$ & \\
\hline 4 & $22(24.7)$ & $26(44.1)$ & \\
\hline Radiation dose [mean (SD)] & $6700.90(431.97)$ & $6885.07(211.52)$ & 0.003 \\
\hline $\begin{array}{l}\text { Follow-up time (months) (median } \\
{[\text { [IQR }] \text { ) }}\end{array}$ & $70.53[28.63,95.40]$ & $63.03[31.30,80.93]$ & 0.357 \\
\hline
\end{tabular}

Bold values denote statistical significance at the $p<0.05$ level $p$ value was calculated using the Pearson $\chi^{2}$ test or Fisher's exact test $C R T$ chemoradiotherapy, $R T$ radiotherapy
The Cox proportional hazards model (Table 4) suggested that age independently predicted poorer OS (HR, 1.07; 95\%CI 1.00-1.15, $p=0.04$ ). Besides, ACCI score higher than 3 , tumor stage and clinical stage were associated with poor prognosis in univariate analysis but were not significantly independent prognostic predictors in multivariate analysis.

\section{Discussion}

It should be noted that elderly NPC patients have been underrepresented in clinical studies for restrict inclusion criteria. Accompanied with poor performance status, multiple comorbidities and decreasing organ function, they tended to be excluded from clinical trials. In MAC meta-analysis, patients older than 60 years old accounted for merely $13 \%$. Moreover, patients older than 70 years old were estimated less than half of that figure [5]. Treatment guidelines were principally tailored for non-elderly patients. Concurrent chemoradiotherapy (CCRT) has been accepted as standard treatment modalities for locally advanced NPC. Nevertheless, it remained unknown whether elderly NPC patients can benefit from chemotherapy while the adding of chemotherapy carries significantly higher rates of acute toxicity. A Meta-analysis of Chemotherapy in Head and Neck Cancer (MACH-NC) found that concomitant chemotherapy brought an absolute benefit of $6.5 \%$ at 5 years and $9.3 \%$ in local control rate, though the effect of chemotherapy decreased with increasing age $(p=0.003)$. Patients aged $>70$ failed to benefit from concomitant chemotherapy [11]. 

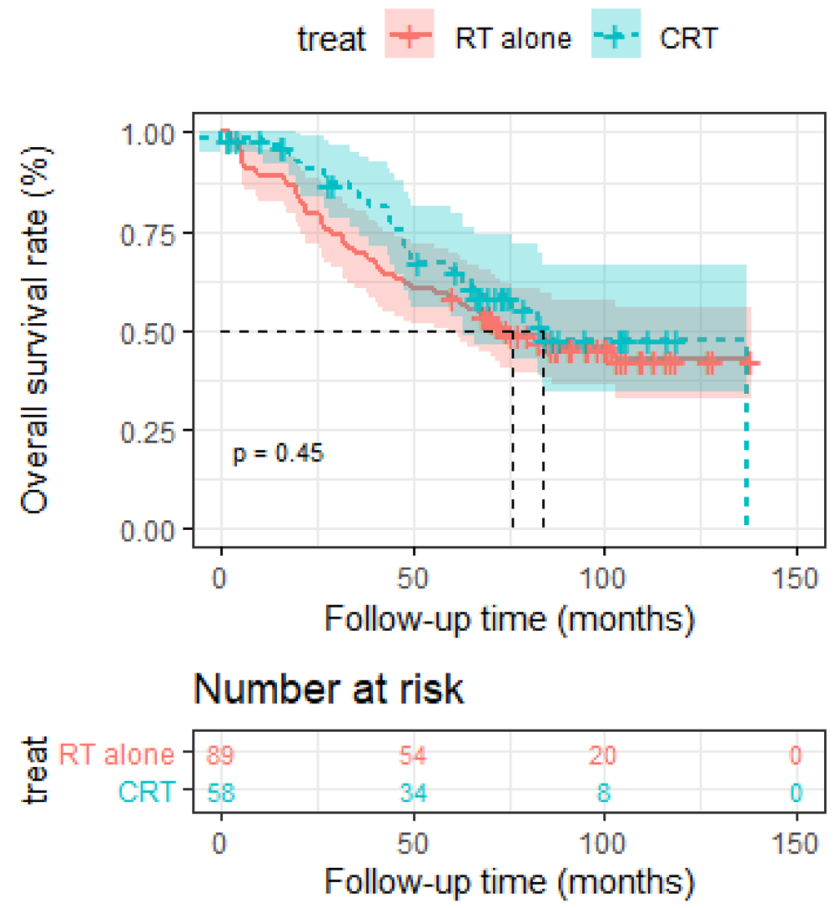

Fig. 1 Kaplan-Meier survival curves between the RT and CRT groups in elderly patients. Shown in results in overall survival (OS). $P$ values were calculated using the unadjusted log-rank test

The cut-off age defining elderly patients varied in different studies. Developed countries have accepted the chronological age of 65 and older as a definition of an elderly population. The National Institute on Aging at the National Institutes of Health classify elderly patients into three categories: young old (65-74 years), older old ( $75-85$ years), and oldest old ( $>85$ years) [12]. With improved physical health of patients, some people who were defined as "old" might be able to withstand aggressive treatment and have favorable prognosis. Patients aged $\geq 70$ years were closely associated with multiple comorbidities, poor performance status and reduced organ reserve, which accounted for their lower tolerance and severe toxicity for chemotherapy $[9,10]$. The reason for choosing 70 as the cut-off points for elderly was that it was close to the threshold in MACH-NC study [11]. Besides, it is often used in head and neck cancer and other type of cancers [13].

In our data, patients receiving radiotherapy alone tended to have older age and more comorbidities. Besides, patients with abnormal hematological abnormalities were less likely to receive chemotherapy at our center considering more treatment-related toxicities. As shown in our data, hematological adverse effects including leukopenia, neutrocytopenia and thrombocytopaenia were significantly higher in CRT group. Elderly patients with comorbidities may be more prone to have severe toxicities, suffer from treatment delays or dose reductions, therefore, they may not survive

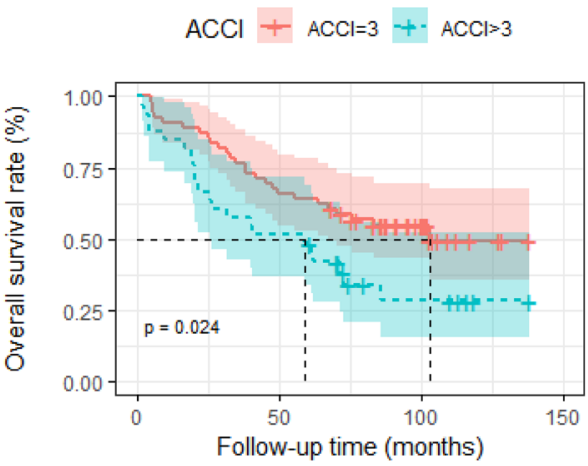

Number at risk
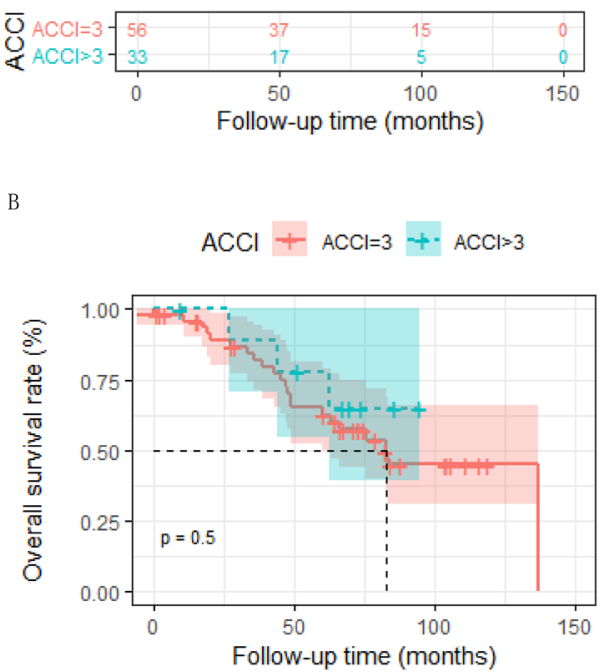

Number at risk
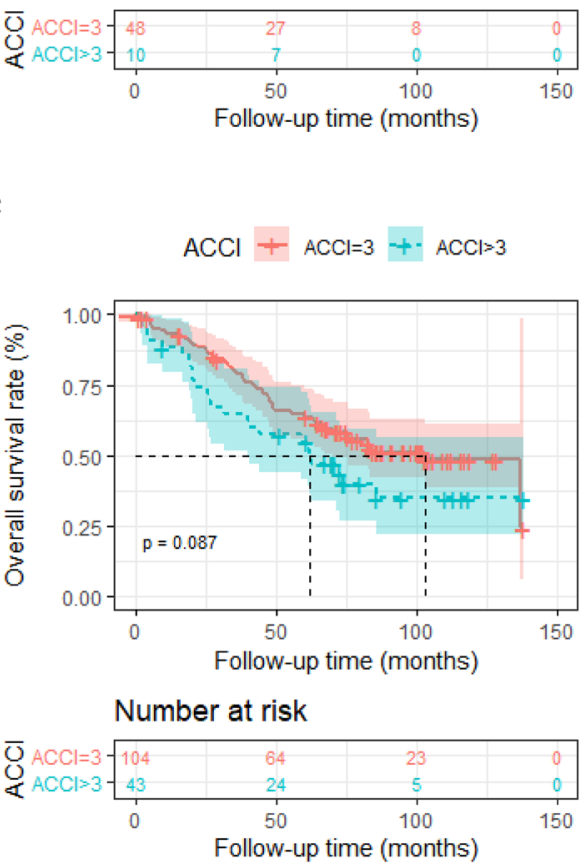

Fig. 2 Kaplan-Meier analysis of overall survival is stratified by ACCI in RT alone group (a), CRT group (b) and the whole group (c). ACCI, age-adjusted Charlson Comorbidity Index 
A

$$
\text { Age }+ \text { Age } s 75-+- \text { Age }>75
$$

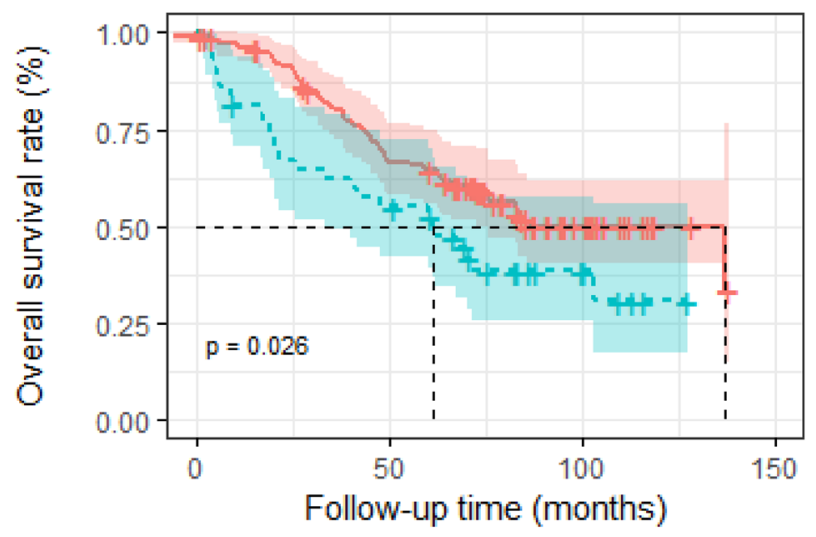

Number at risk

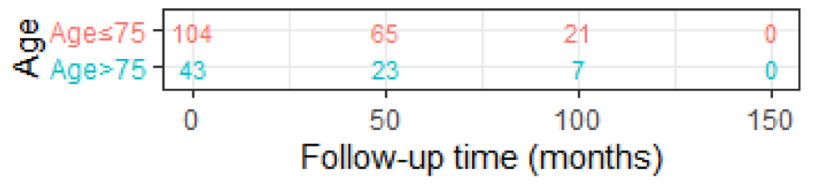

B
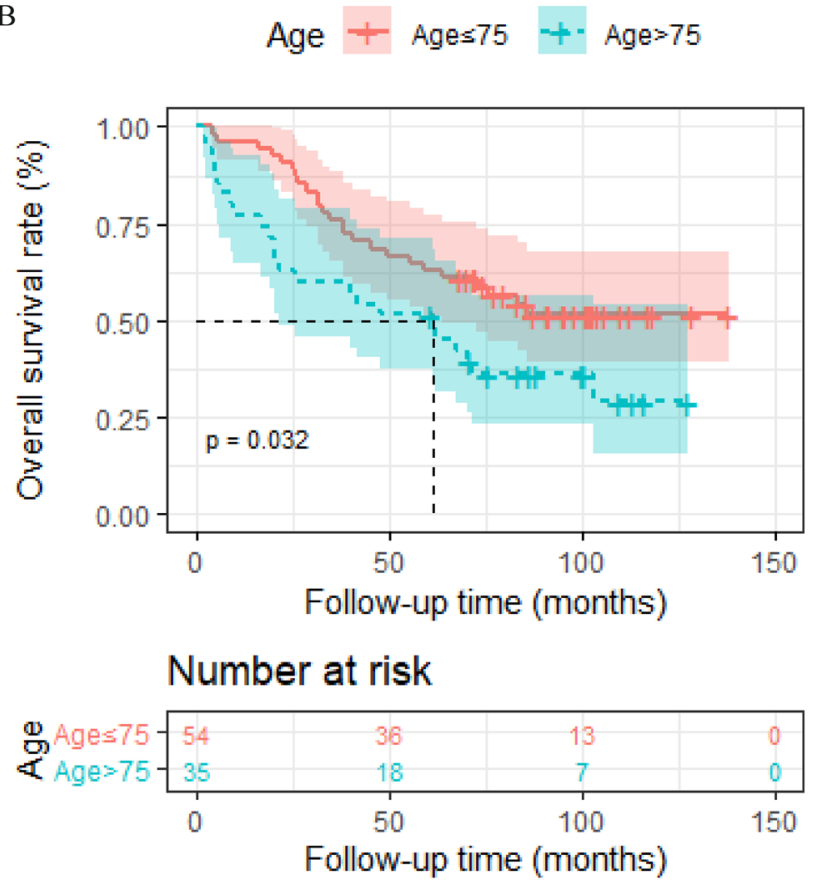

Fig. 3 Kaplan-Meier analysis of overall survival is stratified by age in the entire group (a) and RT alone group (b)

long enough to derive expected benefits from chemotherapy [14].

Two studies demonstrated that chemotherapy improved survival outcome for elderly NPC patients with conventional 2DRT. The 5-year OS rates of the RT alone and
A

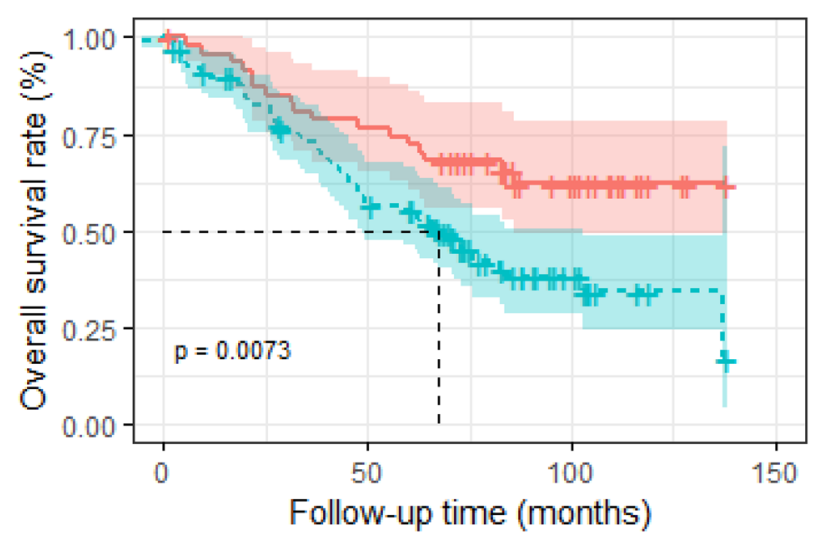

\section{Number at risk}

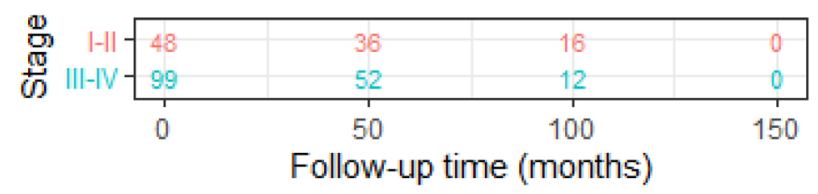

B
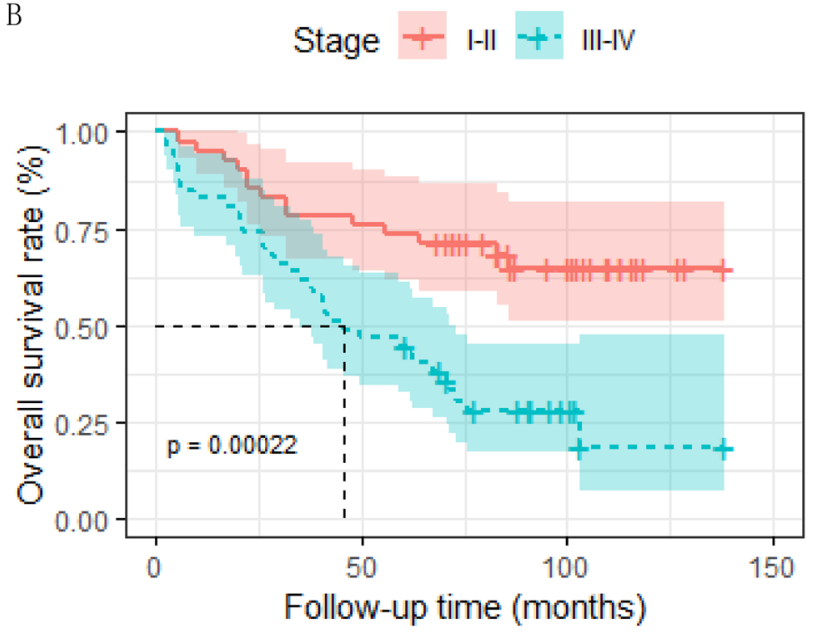

Number at risk

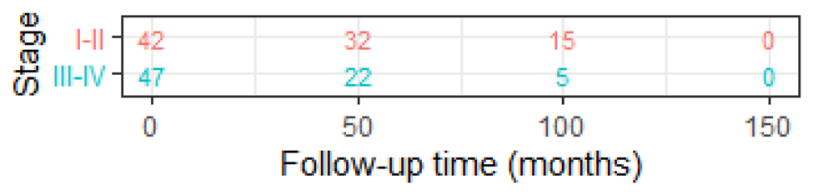

Fig. 4 Kaplan-Meier analysis of overall survival is stratified by stage in the entire group (a) and RT alone group (b)

chemoradiotherapy groups in NPC patients aged $\geq 60$ years reported by Zeng et al. [6] were $40 \%$ and $62 \%(p=0.013)$. Liu et al. made similar conclusion in patients aged $>60$ years receiving conventional radiotherapy [10]. Nonetheless, it was doubted that chemotherapy could bring benefit to geriatric NPC patients with widespread use of IMRT, which 
Table 3 Acute toxicities in RT alone and CRT group

\begin{tabular}{|c|c|c|c|}
\hline Level & RT & CRT & $p$ value \\
\hline$N$ (no. of patients) & 89 & 59 & \\
\hline \multicolumn{4}{|l|}{ Mucositis (\%) } \\
\hline 0 & $19(21.3)$ & $11(18.6)$ & \multirow[t]{5}{*}{0.477} \\
\hline 1 & $19(21.3)$ & $10(16.9)$ & \\
\hline 2 & $29(32.6)$ & $15(25.4)$ & \\
\hline 3 & $20(22.5)$ & $21(35.6)$ & \\
\hline 4 & $2(2.2)$ & $2(3.4)$ & \\
\hline \multicolumn{4}{|l|}{ Dermatitis (\%) } \\
\hline 0 & $49(55.1)$ & $33(55.9)$ & \multirow[t]{5}{*}{0.267} \\
\hline 1 & $18(20.2)$ & $14(23.7)$ & \\
\hline 2 & $17(19.1)$ & $6(10.2)$ & \\
\hline 3 & $3(3.4)$ & $1(1.7)$ & \\
\hline 4 & $2(2.2)$ & $5(8.5)$ & \\
\hline \multicolumn{4}{|l|}{ Emesis (\%) } \\
\hline 0 & $79(88.8)$ & $53(89.8)$ & \multirow[t]{3}{*}{0.847} \\
\hline 1 & $5(5.6)$ & $2(3.4)$ & \\
\hline 2 & $5(5.6)$ & $4(6.8)$ & \\
\hline \multicolumn{4}{|l|}{ Leukopenia (\%) } \\
\hline 0 & $72(80.9)$ & $30(50.8)$ & \multirow[t]{5}{*}{$<0.001$} \\
\hline 1 & $11(12.4)$ & $8(13.6)$ & \\
\hline 2 & $5(5.6)$ & $8(13.6)$ & \\
\hline 3 & $1(1.1)$ & $12(20.3)$ & \\
\hline 4 & $0(0.0)$ & $1(1.7)$ & \\
\hline \multicolumn{4}{|c|}{ Neutrocytopenia (\%) } \\
\hline 0 & $85(95.5)$ & $32(54.2)$ & \multirow[t]{5}{*}{$<0.001$} \\
\hline 1 & $0(0.0)$ & $4(6.8)$ & \\
\hline 2 & $3(3.4)$ & $11(18.6)$ & \\
\hline 3 & $1(1.1)$ & $9(15.3)$ & \\
\hline 4 & $0(0.0)$ & $3(5.1)$ & \\
\hline \multicolumn{4}{|c|}{ Thrombocytopaenia (\%) } \\
\hline 0 & $81(91.0)$ & $38(64.4)$ & \multirow[t]{5}{*}{0.001} \\
\hline 1 & $4(4.5)$ & $10(16.9)$ & \\
\hline 2 & $3(3.4)$ & $6(10.2)$ & \\
\hline 3 & $1(1.1)$ & $4(6.8)$ & \\
\hline 4 & $0(0.0)$ & $1(1.7)$ & \\
\hline \multicolumn{4}{|l|}{ Anemia (\%) } \\
\hline 0 & $82(92.1)$ & $51(86.4)$ & \multirow[t]{4}{*}{0.32} \\
\hline 1 & 7 (7.9) & $6(10.2)$ & \\
\hline 2 & $0(0.0)$ & $1(1.7)$ & \\
\hline 3 & $0(0.0)$ & $1(1.7)$ & \\
\hline
\end{tabular}

Bold values denote statistical significance at the $p<0.05$ level $p$ value was calculated using the Pearson $\chi^{2}$ test or Fisher's exact test $C R T$ chemoradiotherapy, $R T$ radiotherapy

has obtained great local control, better protection for normal organs. In the era of IMRT, whether adding chemotherapy could still benefit elderly individuals remained uncertain. Our data suggested that addition of chemotherapy to IMRT failed to enhance the survival outcomes of patients. The 5-year OS rate of RT alone group was 58.4\% (95\% [CI] 0.490-0.696) compared with 65.2\% (95\% [CI] 0.534-0.796) in CRT group $(p=0.45)$. The survival outcome was comparable to Jin et al.'s study, which failed to confirm the benefit of chemotherapy for elderly NPC patients older than 70 years (5-year OS 56.6-51.2\%, $p=0.617$ ). Moreover, in patients with Adult Comorbidity Evaluation-27 Index (ACE-27) $\geq 2$, chemotherapy was associated with an even inferior OS (26.4\% vs. 50.3\%, $p=0.071)$ [15]. In Sommat et al.'s [16] study, chemotherapy did not bring benefit in stage III and IVA/B patients (aged $\geq 70$ ). In the PSM cohort of Wen et al.'s [17] study (patients over 70 years old), the 3-year CSS was similar in the RT group compared to CRT group $(64.3 \%$ vs. $65.2 \%, p=0.764)$. There was no significant difference in other clinical endpoints assessed, whether chemoradiotherapy could bring benefit to elderly patients with other head and neck cancers worth considering. Recently, Erik Haehl et al. [18] suggested that addition of chemotherapy resulted in a survival benefit for patients aged 65-74 years in the definitive but not in the adjuvant treatment cohort. However, it should be noted that only 4 NPC patients were included in their study group. Besides, the age-dependent outcome in elderly HNSCC patients were similar to our finding that age was an independent prognostic factor.

In terms of toxicity, the incidence rate for severe mucositis and dermatitis (grade 3-4) was $24.7 \%$ and $5.6 \%$ in RT alone group compared to $39 \%$ and $10.2 \%$ in CRT group $(p<0.001)$. Yang et al. [7] suggested severe acute mucositis rates of $16.8 \%$ and $39.8 \%$ in IMRT-alone and IMRT plus CCRT groups $(p<0.001)$. Jin et al. [15] reported an incidence of $18.3 \%$ grade 3-4 mucositis in the entire group. In the PSM cohort of Wen et al.'s [17] study, there was no severe neutropenia and emesis in IMRT-alone group, which was significantly lower than in CCRT group. Thus, IMRT greatly reduced the incidence of severe acute toxicities and increased tolerance to treatment.

Noteworthy, in patient receiving RT only, ACCI score equal to 3 was correlated with superior 5-year OS rate in comparison with higher ACCI score $62.1 \%$ (95\% [CI] $0.510-0.766)$ to $48.5 \%$ (95\% [CI] 0.341-0.689), respectively; $p=0.024)$. ACCI was found to be a prognostic factor for OS in our cohort in the univariate, but not the multivariate analysis. Among the methods quantifying the comorbid ailments, Charlson comorbidity index was used most extensively for its simplicity and straightforward nature. Researches suggested that presence, type and severity of comorbidity condition had an impact on the treatment selection and survival outcome in aged head and neck cancer patients $[19,20]$. Acknowledging the relationship between age and comorbidity in cancer, studies suggest that comorbidity increases with age among head and neck populations $[21,22]$. Due to the importance of age 
Table 4 Cox proportional hazard model

\begin{tabular}{|c|c|c|c|c|}
\hline \multirow[t]{2}{*}{ Variable } & \multicolumn{2}{|l|}{ Univariate analysis } & \multicolumn{2}{|c|}{ Multivariate analysis } \\
\hline & $\mathrm{HR}(95 \% \mathrm{CI})$ & $p$ value & HR $(95 \% \mathrm{CI})$ & $p$ value \\
\hline Age & $1.09(1.02-1.16)$ & 0.01 & $1.07(1.00-1.15)$ & 0.04 \\
\hline Sex (male vs. female) & $1.22(0.69-2.15)$ & 0.499 & NS & NS \\
\hline T category (T1-2 vs. T3-4) & $1.7(1.07-2.7)$ & 0.026 & $1.29(0.72-2.32)$ & 0.39 \\
\hline $\mathrm{N}$ category (N0-1 vs. N2-3) & $1.44(0.9-2.31)$ & 0.131 & NS & NS \\
\hline Overall stage (I-Ii vs. III-IV) & $2.08(1.2-3.59)$ & 0.009 & $1.79(0.90-3.56)$ & 0.10 \\
\hline Radiation dose $(\leq 6600$ vs. $>6600)$ & $1.38(0.87-2.19)$ & 0.175 & NS & NS \\
\hline ACCI score ( $\leq 3$ vs. $>3$ ) & $1.52(0.94-2.47)$ & 0.090 & $1.46(0.86-2.48)$ & 0.16 \\
\hline RT alone vs. CRT & $0.83(0.51-1.35)$ & 0.450 & NS & NS \\
\hline
\end{tabular}

ACCI age-adjusted Charlson Comorbidity Index, AJCC American Joint Commission on Cancer staging, $H R$ hazard ratio, $C I$ confidence interval, $N S$ not significant, $C R T$ chemoradiotherapy, $R T$ radiotherapy on comorbidity, ACCI, the modified CCI, includes age of the patients as correction variable [23]. A population-based study of 4095 NPC patients in Taiwan found that overall survival was significantly associated with the degree of comorbidity and ACCI was a more appropriate prognostic indicator than either original CCI or a revised head and neck comorbidity index (HN-CCI) [24]. Besides, accessing comorbidity level provided information independent from personal performance status. A study of 203 aged cancer patients revealed that the comorbidity condition was not related to performance status [25].

Previous studies reported poor survival for the elderly compared with the younger counterpart [9, 26, 27]. Comorbidity level, DNA copies and other factors, such as CRP and hemoglobin, have been studied for their prognostic value [7, 28]. More potential biomarkers should be studied to select patients who might be fit for chemoradiotherapy. Biological age other than chronological age should be the dominant factor in the selection of best treatment approach for patients with locoregionally confined head and neck cancer.

Integrating these factors into a comprehensive assessment tool might help guide personalized treatment decision and predict survival for elderly NPC patients. A study tried to combine comorbidity level and TNM staging to address this gap [21, 29]. Other factors, such as age, race, gender, socioeconomic status and functional status, might also shed light on clinical decision-making for both health providers and patients.

There are some limitations in our study. First, this study is limited by its retrospective nature, though the prospective trials for the elderly may be hindered by the rarity of patients and the prevalence of comorbidities and decreasing organ function. Second, physical activity assessment, such as Karnofsky performance status (PS), should be concerned, which had been identified as prognostic factor for other head and neck cancers but failed to show significant effect on survival of NPC [30, 31]. Besides, data on chronic toxicities and quality of life were missing in this study. In Jin et al.'s [15] study, four patients
(3.2\%) developed radiation-induced TLI. And our study did not discuss potential biomarkers, such as EBV-DNA, CRP or hemoglobin, which may help select patients for treatment [7, 28]. Another limitation of this study is the small sample size. With the low incidence of NPC in elderly population, this could be acceptable. Therefore, the treatment paradigm and prognostic factors for elderly NPC patients in the era of IMRT warrant further investigation.

In conclusion, our study presented that the survival outcome of patients aged $\geq 70$ years receiving IMRT only was similar to chemoradiotherapy with significantly less acute toxicities. Among the population, age is significantly prognostic for survival outcomes. Further investigation is urgently needed to fully assess the elderly individuals and personalize treatment strategy.

Author contributions YCL and MSN conducted data analysis and drafted the manuscript. FFK, RPZ and CRD participated in data collection. CSH and HMY participated in the design of the study. All authors read and approved the final manuscript.

Funding This work did not receive any specific funding.

Availability of data and materials The datasets used and analyzed during the current study are available from the corresponding author on reasonable request.

\section{Compliance with ethical standards}

Conflict of interest The authors declare that they have no competing interests.

Ethical approval This study was approved by Institutional Review Board of Fudan University Shanghai Cancer Center and was waived of informed consent.

Open Access This article is licensed under a Creative Commons Attribution 4.0 International License, which permits use, sharing, adaptation, distribution and reproduction in any medium or format, as long as you give appropriate credit to the original author(s) and the source, 
provide a link to the Creative Commons licence, and indicate if changes were made. The images or other third party material in this article are included in the article's Creative Commons licence, unless indicated otherwise in a credit line to the material. If material is not included in the article's Creative Commons licence and your intended use is not permitted by statutory regulation or exceeds the permitted use, you will need to obtain permission directly from the copyright holder. To view a copy of this licence, visit http://creativecommons.org/licenses/by/4.0/.

\section{References}

1. Ferlay J, Colombet M, Soerjomataram I et al (2019) Estimating the global cancer incidence and mortality in 2018: GLOBOCAN sources and methods. Int J Cancer 144(8):1941-1953

2. Bray F, Haugen M, Moger TA, Tretli S, Aalen OO, Grotmol $\mathrm{T}$ (2008) Age-incidence curves of nasopharyngeal carcinoma worldwide: bimodality in low-risk populations and aetiologic implications. Cancer Epidemiol Biomarkers Prev 17(9):2356-2365

3. Zhang LF, Li YH, Xie SH et al (2015) Incidence trend of nasopharyngeal carcinoma from 1987 to 2011 in Sihui County, Guangdong Province, South China: an age-period-cohort analysis. Chin J Cancer 34(8):350-357

4. Cooper JS, Pajak TF, Forastiere AA et al (2004) Postoperative concurrent radiotherapy and chemotherapy for high-risk squamous-cell carcinoma of the head and neck. N Engl J Med 350(19):1937-1944

5. Blanchard P, Lee A, Marguet S et al (2015) Chemotherapy and radiotherapy in nasopharyngeal carcinoma: an update of the MAC-NPC meta-analysis. Lancet Oncol 16(6):645-655

6. Zeng Q, Xiang YQ, Wu PH, Lv X, Qian CN, Guo X (2015) A matched cohort study of standard chemo-radiotherapy versus radiotherapy alone in elderly nasopharyngeal carcinoma patients. PLoS ONE 10(3): 0119593

7. Yang Q, Zhao TT, Qiang MY et al (2018) Concurrent chemoradiotherapy versus intensity-modulated radiotherapy alone for elderly nasopharyngeal carcinoma patients with pre-treatment Epstein-Barr virus DNA: a cohort study in an endemic area with long-term follow-up. J Cancer 9(17):3023-3031

8. Verma V, Surkar SM, Moreno AC, Lin C, Simone CB 2nd (2018) Practice patterns and outcomes of chemoradiotherapy versus radiotherapy alone for older patients with nasopharyngeal cancer. Cancer Med 7(5):1604-1611

9. Zhang Y, Yi JL, Huang XD et al (2015) Inherently poor survival of elderly patients with nasopharyngeal carcinoma. Head Neck 37(6):771-776

10. Liu H, Chen QY, Guo L et al (2013) Feasibility and efficacy of chemoradiotherapy for elderly patients with locoregionally advanced nasopharyngeal carcinoma: results from a matched cohort analysis. Radiat Oncol 8:70

11. Pignon JP, le Maitre A, Maillard E, Bourhis J (2009) Metaanalysis of chemotherapy in head and neck cancer (MACH-NC): an update on 93 randomised trials and 17,346 patients. Radiother Oncol 92(1):4-14

12. Parker SL, Tong T, Bolden S, Wingo PA (1997) Cancer statistics, 1997. CA Cancer J Clin 47(1):5-27

13. Gugić J, Strojan P (2012) Squamous cell carcinoma of the head and neck in the elderly. Rep Pract Oncol Radiother 18(1):16-25

14. Lee L, Cheung WY, Atkinson E, Krzyzanowska MK (2011) Impact of comorbidity on chemotherapy use and outcomes in solid tumors: a systematic review. J Clin Oncol 29(1):106-117

15. Jin YN, Zhang WJ, Cai XY et al (2019) The characteristics and survival outcomes in patients aged 70 years and older with nasopharyngeal carcinoma in the intensity-modulated radiotherapy era. Cancer Res Treat 51(1):34-42

16. Sommat K, Yit NLF, Wang F, Lim JHC (2018) Impact of comorbidity on tolerability and survival following curative intent intensity modulated radiotherapy in older patients with nasopharyngeal cancer. J Geriatr Oncol 9(4):352-358

17. Wen YF, Sun XS, Yuan L et al (2019) The impact of Adult Comorbidity Evaluation-27 on the clinical outcome of elderly nasopharyngeal carcinoma patients treated with chemoradiotherapy or radiotherapy: a matched cohort analysis. J Cancer 10(23):5614-5621

18. Haehl E, Ruhle A, David H et al (2020) Radiotherapy for geriatric head-and-neck cancer patients: what is the value of standard treatment in the elderly? Radiat Oncol 15(1):31

19. Derks W, De Leeuw R, Winnubst J, Hordijk GJ (2004) Elderly patients with head and neck cancer: physical, social and psychological aspects after 1 year. Acta Otolaryngol 124(4):509-514

20. Derks W, de Leeuw RJ, Hordijk GJ (2005) Elderly patients with head and neck cancer: the influence of comorbidity on choice of therapy, complication rate, and survival. Curr Opin Otolaryngol Head Neck Surg 13(2):92-96

21. Paleri V, Wight RG, Silver CE et al (2010) Comorbidity in head and neck cancer: a critical appraisal and recommendations for practice. Oral Oncol 46(10):712-719

22. Siddiqui F, Gwede CK (2012) Head and neck cancer in the elderly population. Semin Radiat Oncol 22(4):321-333

23. Charlson M, Szatrowski TP, Peterson J, Gold J (1994) Validation of a combined comorbidity index. J Clin Epidemiol 47(11):1245-1251

24. Yang CC, Chen PC, Hsu CW, Chang SL, Lee CC (2015) Validity of the age-adjusted charlson comorbidity index on clinical outcomes for patients with nasopharyngeal cancer post radiation treatment: a 5-year nationwide cohort study. PLoS ONE 10(1): 0117323

25. Extermann M, Overcash J, Lyman GH, Parr J, Balducci L (1998) Comorbidity and functional status are independent in older cancer patients. J Clin Oncol 16(4):1582-1587

26. Lee AW, Sze WM, Au JS et al (2005) Treatment results for nasopharyngeal carcinoma in the modern era: the Hong Kong experience. Int J Radiat Oncol Biol Phys 61(4):1107-1116

27. Yi JL, Gao L, Huang XD et al (2006) Nasopharyngeal carcinoma treated by radical radiotherapy alone: ten-year experience of a single institution. Int J Radiat Oncol Biol Phys 65(1):161-168

28. Ruhle A, Haehl E, David H et al (2020) The value of laboratory parameters for anemia, renal function, systemic inflammation and nutritional status as predictors for outcome in elderly patients with head-and-neck cancers. Cancers 12(6):19

29. Piccirillo JF, Wells CK, Sasaki CT, Feinstein AR (1994) New clinical severity staging system for cancer of the larynx. Five-year survival rates. Ann Otol Rhinol Laryngol 103(2):83-92

30. Sze HC, Ng WT, Chan OS, Shum TC, Chan LL, Lee AW (2012) Radical radiotherapy for nasopharyngeal carcinoma in elderly patients: the importance of co-morbidity assessment. Oral Oncol 48(2):162-167

31. Rades D, Fehlauer F, Wroblesky J, Albers D, Schild SE, Schmidt R (2007) Prognostic factors in head-and-neck cancer patients treated with surgery followed by intensity-modulated radiotherapy (IMRT), 3D-conformal radiotherapy, or conventional radiotherapy. Oral Oncol 43(6):535-543

Publisher's Note Springer Nature remains neutral with regard to jurisdictional claims in published maps and institutional affiliations. 